\title{
CORRECTION
}

\section{Correction to: Numerical simulation and experimental studies of rock-breaking methods for pre-grooving-assisted disc cutter}

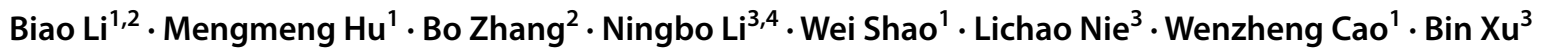

Published online: 2 March 2022

๑) Springer-Verlag GmbH Germany, part of Springer Nature 2022

\section{Correction to: Bulletin of Engineering Geology and the Environment (2022) https://doi.org/10.1007/s10064-022-02594-2}

Originally, Eq. 2 contains an error. This is now correctly presented here.

$$
\begin{aligned}
& F_{N}=F_{T} \cos (\beta) \\
& F_{R}=F_{T} \sin (\beta) \\
& \varphi=\cos ^{-1}\left(\frac{R-P}{R}\right) \\
& \beta=\varphi / 2
\end{aligned}
$$

The original article has been corrected.

The original article can be found online at https://doi.org/10.1007/ s10064-022-02594-2.

Bo Zhang

zhangbo1977@sdu.edu.cn

1 Geotechnical and Structural Engineering Research Center, Shandong University, Jinan 250061, Shandong, China

2 School of Civil Engineering, Shandong University, No.17923 Jingshi Road, Jinan 250061, Shandong, China

3 School of Qilu Transportation, Shandong University, Jinan, China

4 Graduate School of Engineering, Nagasaki University, Nagasaki 852-8521, Japan 